\title{
ALTERNATIVE TECHNIQUE FOR BIODIESEL QUALITY CONTROL USING AN OPTICAL FIBER LONG- PERIOD GRATING SENSOR
}

Rosane Falate

Departamento de Informática, Universidade Estadual de Ponta Grossa, Av. General Carlos Cavalcanti, 4748, 84030-900 Ponta Grossa - PR, Brasil

Karen Nike and Pedro Ramos da Costa Neto

Departamento de Química, Universidade Tecnológica Federal do Paraná, Av. 7 de Setembro 3165, 80230-901 Curitiba - PR, Brasil

Eduardo Cação Jr., Marcia Muller, Hypolito José Kalinowski and José Luís Fabris*

Departamento de Física, Universidade Tecnológica Federal do Paraná, Av. 7 de Setembro 3165, 80230-901 Curitiba - PR, Brasil

Recebido em 12/12/06; aceito em 15/3/07; publicado na web em 17/7/07

\begin{abstract}
Alternative Technique for Biodiesel Quality Control Using an Optical Fiber Long-Period Grating Sensor. We report the use of an optical fiber sensor to measure the soybean oil concentration in samples obtained from the mixture of pure biodiesel and commercial soybean oil. The operation of the device is based on the long-period grating sensitivity to the surrounding medium refractive index, which leads to measurable modifications in the grating transmission spectrum. The proposed analysis method results in errors in the oil concentration of $0.4 \%$ and $2.6 \%$ for pure biodiesel and commercial soybean oil, respectively. Techniques of total glycerol, dynamic viscosity, density, and hydrogen nuclear magnetic resonance spectroscopy were also employed to validate the proposed method.
\end{abstract}

Keywords: biodiesel quality; ethyl esters; optical fiber sensor.

\section{INTRODUCTION}

Methyl and ethyl esters derived from vegetable oils have been receiving considerable attention as alternative sources of renewable energy. A reason for this interest is that fatty ester acids, known as biodiesel, have similar characteristics of petrodiesel oil which allows its use in compression motors without any engine modification ${ }^{1,2}$. Furthermore, numerous studies have shown that the most exhaust emissions resulting from the use of conventional diesel fuel are reduced, with the exception of nitrogen oxides $\mathrm{NO}_{x}$, when biodiesel is adopted ${ }^{3}$.

Biodiesel is produced from vegetable oils in a transesterification process that results in glycerol and fatty acid esters, from the reaction between the triglyceride and an alcohol in the presence of a catalyst. The molecular weight of a typical ester molecule is roughly $1 / 3$ that of a typical triglyceride molecule and therefore it presents a much lower viscosity ${ }^{4,5}$.

The techniques for methyl or ethyl ester contents measurement in the final product of the transesterification process are not simple, affecting its uses in practical applications ${ }^{6}$. Fuel standards deal with quality issues of biodiesel by limiting material contents such as: free and total glycerol (total glycerol is the sum of free glycerol and glycerol bound as mono-, di-, and triacylglycerides), water, free fatty acid and residual alcohol. Compounds concentrations higher than the fuel standards can lead to fuel deterioration during storage, as well as operational problems like, for instance, engine deposits ${ }^{7,8}$.

Techniques such as gas liquid chromatography (GLC), high performance liquid chromatography (HPLC), Hydrogen nuclear magnetic resonance spectroscopy $\left({ }^{1} \mathrm{H} \mathrm{NMR}\right)$, and near infrared (NIR) spectroscopy are used to analyze the final product of the transesterification process, or the biodiesel ${ }^{3,8}$. However, these techniques are rather complicated and expensive to be routinely employed during the biodiesel fabrication process.

*e-mail: fabris@utfpr.edu.br
On the other hand, some results concerning to the use of chemical sensors based on optical fiber have been reported in the past years ${ }^{9,10}$, which present interesting features that contribute to make its use very attractive as chemical sensors. Among these features are immunity to electromagnetic interference, possibility for spectral multiplexing, reduced weight, small volume, high fusion temperature, measurement wavelength encoded, safety at hazardous or explosive environments, possibility for fast or real time, costeffective and quasi-distributed monitoring of chemical processes (with the use of more than one sensor in the same fiber link).

A particular type of optical fiber device, the long-period grating $(\mathrm{LPG})^{11}$, has taken great attention from the researchers, as it presents sensitivity to changes in the surrounding medium refractive index that allows its use as a chemical sensor ${ }^{12}$. This characteristic makes possible the application of LPG sensors in petroleum and natural gas fields ${ }^{13}$ and for environment control ${ }^{14}$.

The LPG is an axially periodic modulation in the optical fiber refractive index with periods of $100-700 \mu \mathrm{m}$, which couples light from the fundamental core propagation mode to co-propagating cladding modes. This mode coupling results in attenuation dips in the fiber transmission spectrum ${ }^{11}$ with central wavelengths given by:

$\lambda_{m}=\left(n_{c o}-n_{c l}^{m}\right) \bullet \Lambda$

where $\lambda_{m}$ is the dip resonance wavelength of the $m$-th cladding mode, $n_{c o}$ and $n_{c l}{ }^{m}$ are the effective refractive indexes of the fundamental core and the $m$-th cladding modes and $\Lambda$ is the grating period. Mechanical stress and temperature changes modify both grating period and mode effective indexes. Moreover, changes in the external medium refractive index also lead to alteration in the cladding mode effective indexes. Such changes result in the wavelength shift of the grating attenuation dips (Equation 1). A particular behavior is observed when the external medium refractive index is higher than the refractive index of the fiber cladding ${ }^{15}$. In such case, the 
wavelength shift is small, but changes in the amplitude of resonance dips are large, and refractive index changes can be detected through intensity measurements in a given spectral interval.

In this work, the intensity changes in the transmission spectrum of a long-period grating were used to detect the soybean oil presence in biodiesel samples and to measure its concentration. For this purpose, thirteen samples with different proportions of pure biodiesel and commercial soybean oil were produced. The obtained results were compared to those ones taken by using ${ }^{1} \mathrm{H}$ NMR and by measuring the refractive index, dynamic viscosity, density and total, free and combined glycerol of the samples.

\section{EXPERIMENTAL}

The ethyl esters of fatty acids were produced using the same procedure of Freedmam ${ }^{16}$ with adaptations. In the transesterification reaction it was used commercial soybean oil (edible-grade oil from Soya ${ }^{\mathrm{tm}}$ ) with an iodine number of 129 (wijs) and a free fatty acid content of $0.049 \%$ (oleic acid). Potassium hydroxide was chosen as catalyst and anhydrous ethanol as the alcohol.

In the procedure used to the transesterification, a solution of potassium hydroxide $(1 \%)$ in anhydrous ethanol was prepared at room temperature and warmed up to $50{ }^{\circ} \mathrm{C}$; after this the soybean oil (12:1 molar ratio) was added to it. The mixture was gently stirred for $1.5 \mathrm{~h}$ and the excess of ethanol was removed, distilled and the glycerol and ester layers were separated by gravity. To check the transesterification process, the ester layer was analyzed using chromatography of the thin layer 94.5:5.0:0.5 (n-hexane/ethyl acetate/acetic acid) with iodine vapour applied as developer. The product of transesterification reaction was cooled and the upper layer (ester) was collected for purification. Such layer was then washed twice with brine $\left(\mathrm{NaCl} 10 \%, 80{ }^{\circ} \mathrm{C}\right)$ and the anhydrous magnesium sulphate was dried over from it. The final product was filtered in a chromatograph column with alternated layers of activated granulate bauxite and starch powder filter ${ }^{17}$.

The experiments were carried out with thirteen samples prepared with different oil mass proportions of commercial soybean oil and pure biodiesel. In our case, the oil mass proportion was determined as a ratio between weights of commercial soybean oil to the resultant sample. The corresponding oil mass proportion in percent values for pure biodiesel and commercial soybean oil were respectively $0.0 \%$ and $100.0 \%$.

The optical fiber sensor used to analyze the characteristics of the prepared samples was a LPG produced by the use of a point-to-point writing method ${ }^{18}$. The LPG was engraved in the optical fiber applying on a bare fiber consecutive and equally spaced arc discharges from a fusion splicer (Siemens, model S46999-M7A-71) resulting in a periodic pattern in the fiber refractive index profile. An advantage of this writing method when compared to the ultraviolet based process is that the produced gratings a higher thermal stability and don't need to be thermally annealed to avoid grating decay. The employed electrical arc parameters were $12 \mathrm{~mA}$ current and $0.5 \mathrm{~s}$ discharge time, used to produce a grating with 65 interaction points and a pitch of $595 \mathrm{~mm}$, with an attenuation band centered at $1544 \mathrm{~nm}$, with FWHM of $15.5 \mathrm{~nm}$ and $7.5 \mathrm{~dB}$ of efficiency in the air.

The experimental set-up used to analyze the samples consists basically of a LPG connected to an optical light source (LED Superlum model 761, $1546.68 \mathrm{~nm}$ central wavelength and $53.12 \mathrm{~nm}$ half bandwidth) and an Optical Spectrum Analyzer Anritsu MS9710B $(0.1 \mathrm{~nm}$ resolution, wavelength stability of $\pm 5 \mathrm{pm}$ and wavelength accuracy of $\pm 0.05 \mathrm{~nm}$ ). To avoid temperature and strain influence on the sensor response, the fiber was fixed with epoxy on a glass plate and the room temperature was kept constant. The sample under analysis was dripped in all LPG surface to guarantee that the sensor was completely covered; then the LPG spectrum was acquired and the sample was removed and inserted in an Abbe refractometer for its refractive index measurement. After the end of each sample measurement, the grating was cleaned with ethanol to remove the residues, allowing the grating to be ready to the next sample analysis.

Measurements of the optical signal intensity in a chosen spectral interval were used for biodiesel analysis since almost all the used samples present refractive indexes higher than the refractive index of the fiber cladding (1.457). Under such conditions, the grating presents a low sensitivity for measurements in the wavelength domain. All the intensities measurements were carried out at the wavelength $1535 \mathrm{~nm}$ within a spectral interval of $0.3 \mathrm{~nm}$, at temperature of $20 \pm 0.5^{\circ} \mathrm{C}$. As transmission spectra can present intensities changes when the measurements are realized under different experimental conditions (for instance, in different days) or due to attenuations in the fiber connections, the intensities were taken relatively to the LED intensity measured under the same conditions (at $1535 \mathrm{~nm}$ and within a $0.3 \mathrm{~nm}$ bandwidth). The ratio between the obtained intensities for the thirteen biodiesel samples to the measured intensities of the LED are used to characterize the biodiesel samples without intensity instability problems. The dynamic viscosity and the ${ }^{1} \mathrm{H}$ NMR analysis of all samples were taken using a Brookfield model LVDVIIICP (CP 42) Viscometer ${ }^{6}$ and a Bruker Advance $200 \mathrm{MHz}$ Spectrometer, using $\mathrm{CDCl}_{3}$ as solvent and TMS as the internal standard, respectively.

The percentage of total glycerol, which is the sum of free glycerol and glycerol bound as mono-, di, and triacylglycerides in the prepared samples was obtained by the official method $\mathrm{Ca}$ 1456 from the American Oil Chemists' Society (A.O.C.S.), known as the Total, Free and Combined Glycerol (Iodometric-Periodic Acid) Method $^{19}$. In this method, total glycerol is determined by saponification of the oil that remains after the transesterification and then by reacting the glycerol produced with periodic acid. The resulting compound is titrated with normalized sodium thiosulfate solution using a starch indicator solution.

\section{RESULTS AND DISCUSSION}

The glycerol percentages in the samples were obtained with the Ca 14-56 method and are shown in the Figure 1.

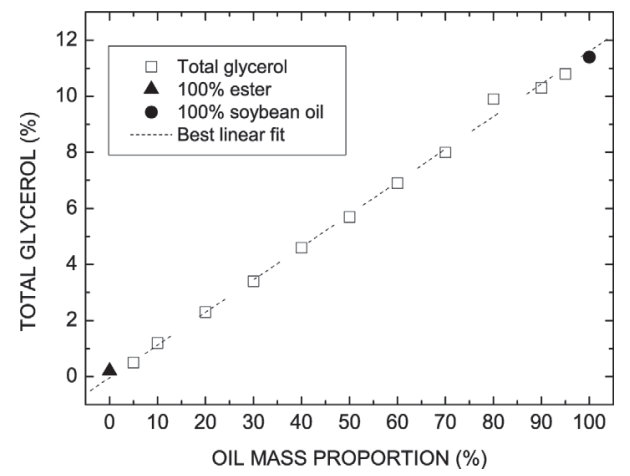

Figure 1. Total glycerol of the 13 samples with oil mass proportion from 0 to $100 \%$, including the pure biodiesel sample ( $\mathbf{\Delta})$ and commercial soybean oil (

As expected, the higher the soybean oil contents in the sample, the higher the total glycerol content. For the commercial soybean oil and biodiesel samples, the total glycerol percentages were $11.4 \%$ and $0.2 \%$, respectively. The best linear fit presented a linear coefficient of -0.041 and an angular coefficient of 0.117 with a correlation coefficient of 0.9982 . For the sake of comparison, we 
also determined the dynamic viscosity, density, and refractive index of the prepared samples (Figure 2a, b). As expected, samples with lower oil contents present lower dynamic viscosity, density, total glycerol, and refractive index values. This behavior reflects in an increase of the molecular weight, polarity, steric hindrance and intermolecular forces. The obtained values are in agreement with those previously reported for ethyl esters ${ }^{6,20}$. The lower and higher refractive index values of the analyzed samples are respectively 1.4576 for pure biodiesel and 1.4737 for commercial soybean oil.

Figure 2(c) also shows that, apart from the lowest value of used oil mass proportions, total glycerol dependence on refractive index of the samples can be approximated to a linear behavior. This functional dependence is the key that allows using the proposed sensor for the biodiesel analysis. The angular coefficient obtained was $700.93 \pm 13.66 \% / \mathrm{RIU}$, that represents the total glycerol percentage per refractive index units with a correlation coefficient of 0.9979 .
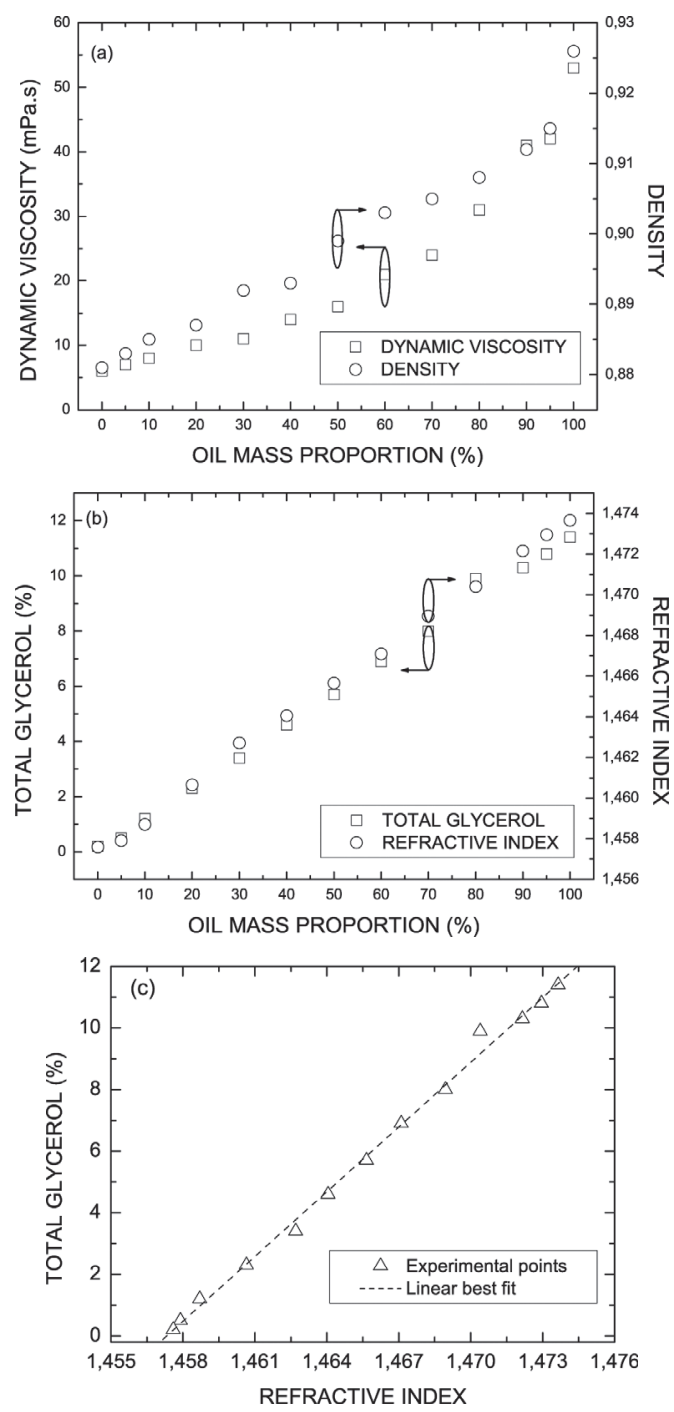

Figure 2. (a) Dynamic viscosity and (b) density of the 13 samples with oil mass proportion from 0 to $100 \%$ and (c) relation between the total glycerol and the refractive index of the samples

The thirteen samples were put in contact with the fiber sensor and some of the results for the LPG transmission spectrum are shown if Figure 3. As the refractive index of the samples are higher than the LPG cladding refractive index, the shifts in the resonance wavelength are lower than the shifts when the grating is immersed in a fluid whose refractive index is lower than the cladding refractive index. The most pronounced effect is the change in the LPG transmission intensity ${ }^{12}$; however, the resonance wavelength shift must still be considered, and changes in the measured intensities are caused by both the variation in the depth and in the resonance wavelength.
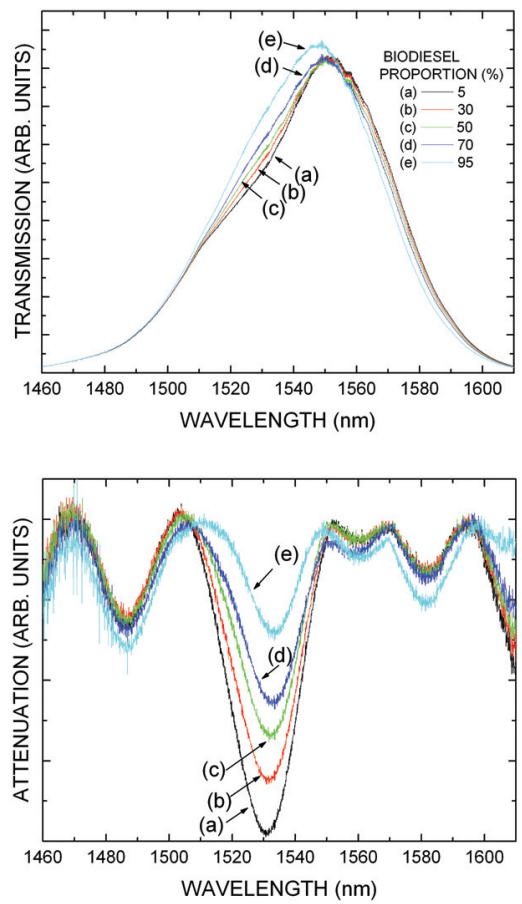

Figure 3. (a) LPG transmission spectra and (b) LPG attenuation dips for five different biodiesel proportions in the samples

The results for the measured intensity ratio are shown in Figure 4. The optical fiber sensor response shows an exponential behavior with the increase in the oil mass proportion in the biodiesel samples and an empirical adjust of the experimental points was provided by a first order exponential curve (continuous line). The obtained exponential function (solid line) is given by:

$I(x)=a+b \cdot \exp (c . x)$

In Equation (2), $x$ is the soybean oil mass proportion, $a=0.2660 \pm 0.0027, b=0.0822 \pm 0.0024$, and $c=-0.0198 \pm 0.0015$ are constant numbers. The correlation coefficient $\left(r^{2}\right)$ is 0.9968 .

The used fitting allows finding the soybean oil concentration in one particular sample and thereby represents the sensor calibration curve for this type of fluid. By using the exponential function, it is possible to determine the system resolution. Considering the worst case in the experiment, the minimum intensity variation was 0.0006 for the soybean oil mass proportion in the range from $95 \%$ to $100 \%$. Such intensity variation represents an error in the obtained oil mass proportion for pure biodiesel and commercial soybean oil of $0.4 \%$ and $2.6 \%$ respectively.

The results of the samples analysis by ${ }^{1} \mathrm{H}$ NMR are also shown in Figure 4. In this technique the oil content is given by the ratio between the peak areas of glycerol methylenic (4 hydrogen in the two methylene groups of triacylglycerol moiety) and ester ethoxy, in the region of $4.05-4.40 \mathrm{ppm}^{21}$. The experimental points were also adjusted by a first order exponential curve (doted line, Equation 2 ), with $a=0.213 \pm 0.060, b=0.227 \pm 0.049$, and $c=0.0169 \pm 0.0018$. The correlation coefficient is 0.9955 . 


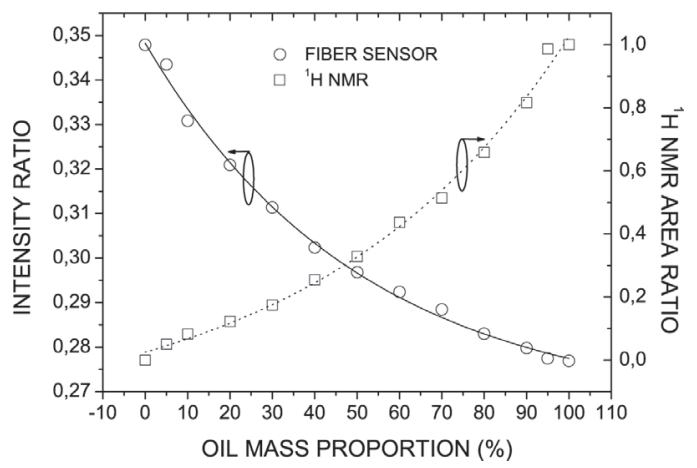

Figure 4. Optical fiber sensor response and ${ }^{1} \mathrm{H} N M R$ area ratio to the oil mass proportion in biodiesel samples

\section{CONCLUSION}

The sensitivity of a long-period grating to external medium refractive index changes was employed to determine the presence and content of soybean oil in biodiesel. Samples analysis using total glycerol, relative density, dynamic viscosity, ${ }^{1} \mathrm{H}$ NMR compared with the results presented by the fiber sensor shows that the refractive index can be used as a measurand of soybean oil content in biodiesel. Two results validate the use of the LPG sensor for this purpose: refractive index and total glycerol of the samples have a linear dependence, and LPG transmission spectra intensity presents a well-defined function for the sample refractive index.

The LPG response to the sample refractive index and, consequently, to the oil mass proportion in the biodiesel samples results in errors in the determination of this parameter of about $0.4 \%$ and $2.6 \%$ for pure biodiesel and commercial soybean oil samples, respectively.

Besides of the high sensitivity to refractive index changes, LPG is an in-fiber compact and cheap device that presents real time response, and can be easily integrated in an optical link. In this way, the biodiesel quality can be efficiently verified without the necessity of dislocations to a specific laboratory. In addition, by measuring the LPG response in the intensity domain instead of the frequency domain, the OSA can be replaced by a more simple and suitable instrumentation for an industrial process. On the other hand, although LPG sensor was proposed to determine the soybean oil concentration in biodiesel, this sensor can be also applied to monitor the biodiesel production process providing a product with reduced costs in a minor time interval.

\section{ACKNOWLEDGMENT}

This work was partially done under the auspices of CAPES, CNPq, and Fundação Araucária (Brazilian agencies). The authors thank to Federal University of Santa Catarina, where the ${ }^{1} \mathrm{H}$ NMR analysis were taken.

\section{REFERENCES}

1. Schucharddt, U.; Serchel, R.; Vargas, R. M.; J. Braz. Chem. Soc. 1998, 9, 199.

2. Costa Neto, P. R.; Rossi, L. F. S.; Zagonel, G. F.; Ramos, L. P.; Quim. Nova 2000, 23, 531

3. Gelbard, G.; Bres, O.; Vargas, R. M.; Schucharddt, U.; J. Am. Oil Chem. Soc. 1995, 72, 1239

4. Clark, S. J.; Wagner, L.; Schrock, M. D.; Piennaar, P. G.; J. Am. Oil Chem. Soc. 1984, 61, 1632

5. Knothe, G.; Bagby, M. O.; Ryan, T. W.; J. Am. Oil Chem. Soc. 1998, 75, 1007.

6. Filippis, D. P.; Giavarini, C.; Scarsella, M.; Sorrentino, M.; J. Am. Oil Chem. Soc. 1995, 72, 1399.

7. Knothe, G.; J. Am. Oil Chem. Soc. 1999, 76, 795.

8. Knothe, G.; J. Am. Oil Chem. Soc. 2000, 77, 489.

9. Wolfbeis O. S.; Anal. Chem. 2004, 76, 3269.

10. Lee, B.; Opt. Fiber Technol. 2003, 9, 57.

11. Vengsarkar, A. M.; Lemaire, P. J.; Judkins, J. B.; Bhatia, V.; Erdogan, T.; Sipe, J.E. ; J. Lightwave Technol. 1996, 14, 58.

12. Patrick, H. J.; Kersey, A. D.; Bucholtz, F.; J. Lightwave Technol. 1998, 16, 1606.

13. Falate, R.; Kamikawachi, R. C.; Muller, M.; Kalinowski, H. J.; Fabris, J. L.; Sens. Actuators, B 2005, 15, 430.

14. Brambilla, G.; Kee, H. H.; Pruneri, V.; Newson, T. P.; Opt. Lasers Eng. 2002, 37, 215.

15. Lee, B. H.; Liu, Y.; Lee, S. B.; Choi S. S.; Opt. Lett. 1997, 22, 1769

16. Freedman, B.; J. Am. Oil Chem. Soc. 1984, 61, 1638.

17. Yates, R. A.; Caldwell, J. D.; J. Am. Oil Chem. Soc. 1993, 70, 507.

18. Rego, G.; Okhotnikov, O.; Dianov, E.; Sulimov, V.; J. Lightwave Technol. 2001, 19, 1574.

19. Canakci, M.; van Gerpen, J.; Am. Soc. Agric. Eng. 1999, 42, 1203.

20. Tat, M. E.; Gerpen, J. H. V.; J. Am. Oil Chem. Soc. 1998, 76, 1511.

21. Costa Neto, P. R.; Caro, M. S. B.; Mazzuco, L. M.; Nascimento, M. G.; J. Am. Oil Chem. Soc. 2004, 81, 1111. 